\title{
IMPULSIVE RESPONSE IN CENTRIFUGAL LIQUID CHROMATOGRAPHY
}

\author{
ATSUSHI ENDO, NOBORU SUZUKI, SHINJI HAGIWARA, \\ MITSUHIKO UMEMURA AND HIROSHI UTSUGI \\ Department of Environmental Chemistry, Utsunomiya University, Utsunomiya 321 \\ TOSAO FUKUDA \\ Hitachi Koki Co., Ltd., Katsuta 312
}

\begin{abstract}
Key Words: Centrifugal Liquid Chromatography, Mass Transfer, Impulse Response, Adsorption, Disk Type Column
\end{abstract}

\section{Introduction}

Centrifugal liquid chromatography is a kind of separation system which uses the difference of adsorptivity of solutes on packing particles. The liquid is forced to flow through a disc-type column by centrifugal force created by the rotation of the disc column. Since the rate of revolution determines the flow rate of the liquid, rapid revolution gives a high flow rate. Thus centrifugal liquid chromatography is useful for treating and fractioning large amounts of liquid. The mechanism of the separation process in centrifugal liquid chromatography was investigated by using dyes as a tracer for porous silica gel. ${ }^{3)}$ In this theoretical analysis, impulse response was calculated and the calculated chromatographic curve was compared with experimental ones.

\section{Experimental Apparatus and Procedure}

Figure 1 shows a schematic illustration of a centrifugal liquid chromatograph (CLC-3 type, made by Hitachi Koki). The diameter of the disc-type column is $0.27 \mathrm{~m}$. The column thickness is $0.003 \mathrm{~m}$, controllable by a spacer. Toluene (1st class, $99 \mathrm{vol} \%$, made by Kanto Chemical), used as an eluant, is introduced from the tank to the inlet of the apparatus, then flows through the rotating disc-type column. The flow rate can be changed by changing the revolution rate. The dye used as a tracer is "Oil Yellow". Its molecular formula is $\mathrm{C}_{17} \mathrm{H}_{15} \mathrm{~N}_{3}$. The dye is injected into the toluene flow by a whole pipette. This tracer enters at entrance $r=r_{1}$, then flows through the entire length of a column, $r_{2}-r_{1}$. The effluent from the outlet is led to an ultraviolet light monitor and the concentration of tracer in the effluent is determined. Chromatographic curve peaks are obtained from a recorder.

Received August 31, 1983. Correspondence concerning this article should be addressed to A. Endo.
The particles used as adsorbent are porous silica gel (for chromatography, made by Kanto Chemical), with the physical properties listed in Table 1. It is shown that the larger-particle silica gel has the larger specific surface area.

Adsorption isotherms at $298 \mathrm{~K}$ were obtained from the equilibrium concentrations, which were determined with a spectrophotometer after 24 hours of stirring. The relation between adsorbed amount and equilibrium concentration of the dye is shown in Fig. 2. In the range of low concentration the relation is linear and the difference of specific surface area appears in the difference of saturation concentrations.

The flow pattern in a rotating column can be seen from the photograph, which was taken by using a stroboscope. It is recognized from this photograph that the direction of liquid flow in a rotating column is radial only and that no spiral flow exists.

\section{Mathematical Analysis and Discussion}

The concentration, $C(r, t)$, of the adsorbing species

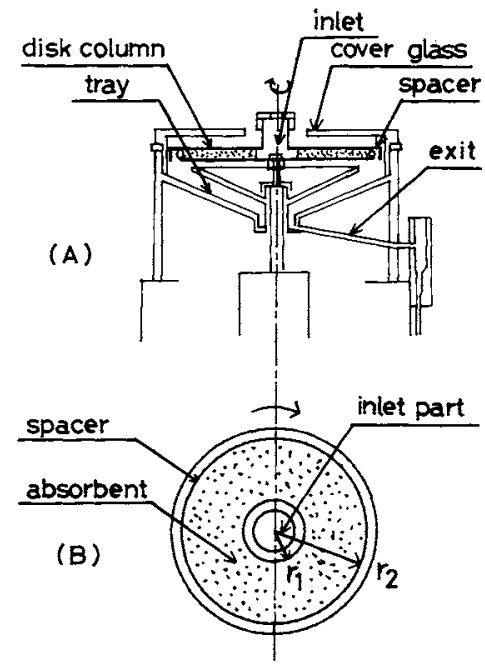

Fig. 1. Apparatus of centrifugal liquid chromatography. 
Table 1. Physical properties of adsorbent (silica gel)

\begin{tabular}{lcccc}
\hline Adsorbent & $\begin{array}{c}\text { Particle } \\
\text { radius } \\
R \times 10^{3}[\mathrm{~m}]\end{array}$ & $\begin{array}{c}\text { Particle } \\
\text { density } \\
\rho_{p}\left[\mathrm{~kg} / \mathrm{m}^{3}\right]\end{array}$ & $\begin{array}{c}\text { Apparent } \\
\text { density } \\
\rho\left[\mathrm{kg} / \mathrm{m}^{3}\right]\end{array}$ & $\begin{array}{c}\text { Specific } \\
\text { surface area } \\
{\left[\mathrm{m}^{2} / \mathrm{g}\right]}\end{array}$ \\
\hline 20-40 mesh & $0.32 \pm 0.12$ & 2400 & 480 & 476 \\
$40-60$ mesh & $0.16 \pm 0.04$ & 2400 & 540 & 410 \\
\hline
\end{tabular}

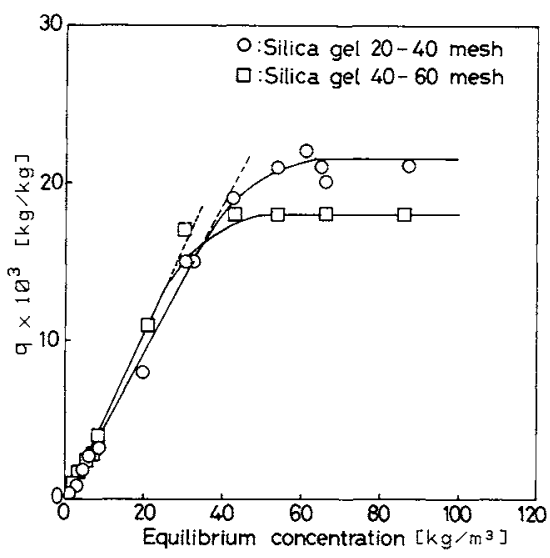

Fig. 2. Adsorption isotherms of Oil Yellow for various silica gels.

as a function of time and radial position in a rotating column can be written as the following set of differential equations:

The mass balance of the adsorbable component in the liquid phase (taking account of radial flow convection, dispersion and adsorption)

$$
\begin{aligned}
\frac{\partial^{2} C}{\partial r^{2}}+ & \frac{1}{r}\left(1-\frac{Q}{2 \pi h \varepsilon_{b} D}\right) \frac{\partial C}{\partial r} \\
& -\frac{3\left(1-\varepsilon_{b}\right)}{R \varepsilon_{b}} \frac{D_{e}}{D}\left(\frac{\partial C_{p}}{\partial r_{p}}\right)_{R}=\frac{1}{D} \frac{\partial C}{\partial t},
\end{aligned}
$$

the mass balance of this component in the particle which is assumed to be a sphere

$$
D_{e}\left(\frac{\partial^{2} C_{p}}{\partial r_{p}^{2}}+\frac{2}{r_{p}} \frac{\partial C_{p}}{\partial r_{p}}\right)-\rho_{p} \frac{\partial C_{a}}{\partial t}=\varepsilon_{p} \frac{\partial C_{p}}{\partial t}
$$

and the adsorption rate which is assumed to be proportional to the difference between the concentration in the particle and the equilibrium concentration

$$
\frac{\partial C_{a}}{\partial t}=k_{a}\left(C_{p}-\frac{C_{a}}{K_{a}}\right)
$$

where the boundary condition of mass transfer at the particle surface is given as

$$
D_{e}\left(\frac{\partial C_{p}}{\partial r_{p}}\right)_{R}=k_{f}\left\{C-\left(C_{p}\right)_{R}\right\}
$$

while other boundary conditions are

$$
\begin{array}{lll}
C=\delta(t) & \text { at } & r=r_{1} \\
C=0 & \text { at } & r=\infty \\
\partial C_{p} / \partial r_{p}=0 & \text { at } & r_{p}=0
\end{array}
$$

and initial conditions

$$
\begin{array}{lll}
C=0 & \text { at } & t=0 \\
C_{p}=0 & \text { at } & t=0 \\
C_{a}=0 & \text { at } & t=0
\end{array}
$$

Taking the Laplace transform on $t$, and solving the resulting ordinary differential equations for $\bar{C}(s)$ gives the transfer function for the outlet of the column $r=r_{2}$ as follows:

$$
F(s)=\left(\frac{r_{2}}{r_{1}}\right)^{v} \frac{I_{-v}\left(\kappa r_{2}\right)}{I_{-v}\left(\kappa r_{1}\right)}
$$

where

$$
\begin{aligned}
& \nu=Q / 4 \pi h \varepsilon_{b} D \\
& \kappa=\sqrt{\frac{S}{D}+\mu} \\
& \mu=\frac{3\left(1-\varepsilon_{b}\right)}{\varepsilon_{b}} \frac{D_{e}}{R^{2} D} \frac{1}{\frac{D_{e}}{R k_{f}}+\frac{1}{R \sqrt{\lambda} \operatorname{coth}(R \sqrt{\lambda})-1}} \\
& \lambda=\frac{S}{D_{e}}\left(\varepsilon_{p}+\frac{\rho_{p} k_{a}}{S+k_{a} / K_{a}}\right)
\end{aligned}
$$

It is impossible to obtain impulse response analytically because the inverse Laplace transform of Eq. (7) is unknown. Therefore, impulse response $Q_{\delta}(t)$ is evaluated numerically by using the real part of the frequency response ${ }^{1)}$ which is obtained by replacing complex $S$ by $i \omega$.

$$
\left.O_{\delta}(t)=\frac{2}{\pi} \int_{0}^{\infty} \operatorname{Real}(F(i \omega))\right) \cos \omega t d \omega
$$

To compare the calculated peak shape of the impulse injection with the experimental peak shape, measurements were made of the pulse response in our apparatus using a column packed with $R=0.32 \times$ $10^{-3}$ or $0.16 \times 10^{-3} \mathrm{~m}$ silica gel. Column porosity was about 0.79 . Measurements were made at different flow rates. The range of rotation of the column was from $1.5-2 \mathrm{~s}^{-1} .1 \times 10^{-6} \mathrm{~m}^{3}$ of $1 \times 10^{-3} \mathrm{~kg} / \mathrm{m}^{3}$ solution was injected into the inlet of the rotating column $r=r_{1}$ as an impulse signal.

Adsorption equilibrium constant $K_{a}$ was determined from the initial inclination of the adsorption isotherm shown in Fig. 2. Dispersion coefficient $D$ was estimated from the relation $P e=(2 R) u / D=$ const. which was estimated from the figure concerning the dispersion in a packed tower, presented by Suzuki. $\left.{ }^{2}\right)$ 
Mass transfer coefficient $k_{f}$ is obtained from Wilson's equation. ${ }^{5}$

$$
0.0016<R e_{p}<55 \quad \varepsilon_{b} j_{D}=1.09 R e_{p}^{-2 / 3}
$$

Molecular diffusion coefficient $D_{i}$ is estimated from Wilke's equation. ${ }^{4)}$ Effective diffusion coefficient $D_{e}$ is estimated from the following relation:

$$
D_{e}=\varepsilon_{p} D_{i} / \tau
$$

where tortuosity $\tau$ is assumed to be $\sqrt{2}$. Adsorption rate constant $k_{a}$ is so large that it may be substantially assumed as $k_{a}=\infty$. Flow rate $Q$ is measured by using a measuring cylinder.

On the basis of the facts above mentioned, the theoretical response was calculated. The shape of the calculated impulse response seems to be more sensitive to the values of adsorption equilibrium constant $K_{a}$ and dispersion coefficient $D$ than other parameters.

Figures 3 and 4 compare the shapes of the experimental and the calculated chromatographic curves. The calculated impulse responses show a good approximation to the experimental curves except in a tailing zone. These results show that determinations of the values of adsorption equilibrium constant and dispersion coefficient are reasonable. It is also clear from Figs. 3 and 4 that difference in particle diameter gives a remarkable difference in the values of dispersion coefficients. Generally speaking, the experimental injection is not made instantaneously because a certain period of time is necessary for the dye to enter the column. ${ }^{4)}$ Therefore the distortion of this input signal has a little influence on the differences of the chromatographic curves in Figs. 3 and 4.

$\begin{array}{llr}\text { Nomenclature } & \\ C & =\text { concentration in flow } & {\left[\mathrm{kg} / \mathrm{m}^{3}\right]} \\ \tilde{C} & =\text { Laplace transform of } C & \\ C_{a} & =\text { concentration in adsorbent } & {[\mathrm{kg} / \mathrm{kg}]} \\ C_{p} & =\text { concentration in particle } & {\left[\mathrm{kg} / \mathrm{m}^{3}\right]} \\ D & =\text { dispersion coefficient } & {\left[\mathrm{m}^{2} / \mathrm{s}\right]} \\ D_{e} & =\text { effective diffusion coefficient } & {\left[\mathrm{m}^{2} / \mathrm{s}\right]} \\ F & =\text { transfer function } & \\ h & =\text { column thickness } & {[\mathrm{m}]} \\ I_{v} & =\text { first-kind modified Bessel function of order } v \\ j_{D} & =j \text { factor } & {[-]} \\ k_{a} & =\text { adsorption rate constant } & {\left[\mathrm{m}^{3} / \mathrm{kg} \cdot \mathrm{s}\right]} \\ K_{\mathrm{a}} & =\text { adsorption equilibrium constant } & {\left[\mathrm{m}^{3} / \mathrm{kg}\right]} \\ N & =\text { revolution rate } & {[\mathrm{s}-1]} \\ q & =\text { concentration in adsorbent at equilibrium } & {[\mathrm{kg} / \mathrm{kg}]} \\ Q & =\text { flow rate } & {[\mathrm{m} 3 / \mathrm{s}]} \\ r & =\text { radius in disc column } & {[\mathrm{m}]} \\ r_{p} & =\text { radius in particle } & {[\mathrm{m}]} \\ R & =\text { outer radius of particle } & {[\mathrm{m}]} \\ R e_{p} & =\text { Reynold's number } & {[-]} \\ s & =\text { complex } & \\ t & =\text { time } & {[\mathrm{s}]}\end{array}$

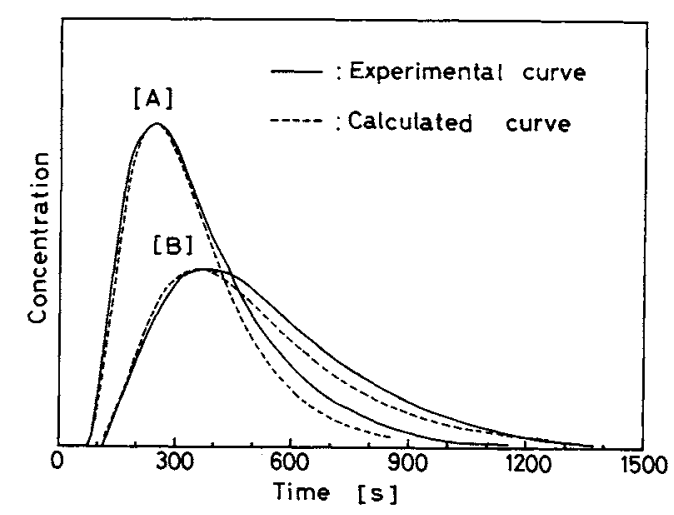

Fig. 3. Comparison of experimental and calculated chromatographic curves for silica gel (20-40 mesh).

[A]: $v=9.0, Q=0.50 \times 10^{-6} \mathrm{~m}^{3} / \mathrm{s}, \quad R=0.32 \times 10^{-3} \mathrm{~m}, \quad h=$ $3 \times 10^{-3} \mathrm{~m}, R e_{p}=0.4, \varepsilon_{b}=0.80, \quad D=1.8 \times 10^{-6} \mathrm{~m}^{2} / \mathrm{s}, \quad k_{f}=$ $4.1 \times 10^{-4} \mathrm{~m} / \mathrm{s}, K_{a}=0.46 \times 10^{-3} \mathrm{~m}^{3} / \mathrm{kg}, D_{i}=1.24 \times 10^{-9} \mathrm{~m}^{2} / \mathrm{s}$, $N=1.8 \mathrm{~s}^{-1}$.

[B]: $\quad v=7.0, \quad Q=0.34 \times 10^{-6} \mathrm{~m}^{3} / \mathrm{s}, \quad R=0.32 \times 10^{-3} \mathrm{~m}, \quad h=$ $3 \times 10^{-3} \mathrm{~m}, R e_{p}=0.3, \varepsilon_{b}=0.80, \quad D=1.5 \times 10^{-6} \mathrm{~m}^{2} / \mathrm{s}, \quad k_{f}=$ $1.6 \times 10^{-4} \mathrm{~m} / \mathrm{s}, K_{a}=0.46 \times 10^{-3} \mathrm{~m}^{3} / \mathrm{kg}, D_{i}=1.24 \times 10^{-9} \mathrm{~m}^{2} / \mathrm{s}$, $N=1.5 \mathrm{~s}^{-1}$.

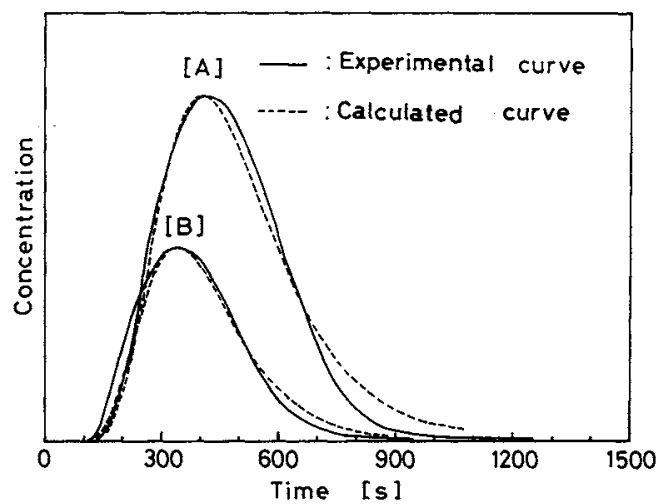

Fig. 4. Comparison of experimental and calculated chromatographic curves for silica gel (40-60 mesh). [A]: $v=12.0, Q=0.35 \times 10^{-6} \mathrm{~m}^{3} / \mathrm{s}, \quad R=0.16 \times 10^{-3} \mathrm{~m}, h=$ $3 \times 10^{-3} \mathrm{~m}, R e_{p}=0.2, \varepsilon_{b}=0.8, D=9.7 \times 10^{-7} \mathrm{~m}^{2} / \mathrm{s}, k_{f}=2.6 \times$ $10^{-4} \mathrm{~m} / \mathrm{s}, K_{a}=0.53 \times 10^{-3} \mathrm{~m}^{3} / \mathrm{kg}, D_{i}=1.24 \times 10^{-9} \mathrm{~m}^{2} / \mathrm{s}, N=$ $1.7 \mathrm{~s}^{-1}$.

[B]: $v=13.0, Q=0.42 \times 10^{-6} \mathrm{~m}^{3} / \mathrm{s}, \quad R=0.16 \times 10^{-3} \mathrm{~m}, h=$ $3 \times 10^{-3} \mathrm{~m}, R e_{p}=0.2, \varepsilon_{b}=0.77, D=1.07 \times 10^{-6} \mathrm{~m}^{2} / \mathrm{s}, \quad k_{f}=$ $2.7 \times 10^{-4} \mathrm{~m} / \mathrm{s}, K_{a}=0.53 \times 10^{-3} \mathrm{~m}^{3} / \mathrm{kg}, D_{i}=1.24 \times 10^{-9} \mathrm{~m}^{2} / \mathrm{s}$, $N=1.7 \mathrm{~s}^{-1}$.

$$
\begin{array}{lr}
=\text { Dirac's delta function } \\
=\text { porosity of disc column } \\
=\text { porosity of particle }
\end{array}
$$

\section{Literature Cited}

1) Izawa, K.: "Introduction to Automatic Control," Elsevier (1963).

2) Suzuki, M.: Kagaku Kogaku, 29, 253 (1965).

3) Utsugi, H., A. Endo, N. Suzuki, S. Hagiwara, M. Umemura 
and T. Fukuda: J. Japan Soc. Color Material (Shikizai), 56, 787 (1982).

4) Wakao, N., K. Tanaka and H. Nagai: Chem. Engng. Sci., 31 (1976).

5) Wilke and Chang: Am. Inst. Chem. Engrs. J., 1, 264 (1955).
6) Wilson, E. J. and C. G. Geankoplis: Ind. Eng. Chem., Fundam., 5, 9 (1966).

(Presented at PACHEC '83, Korea at Scoul, May 10, 1983.)

\title{
ENERGY EFFICIENCY OF ELECTROSTATIC TYPE SMALL BUBBLE GENERATOR
}

KAZUSHI TAN AND SUMITOSHI OGATA

Department of Chemical Engineering, Kyushu University, Fukuoka 812

\begin{abstract}
Key Words: Electrochemical Engineering, Bubble Size Reduction, Electrohydrodynamic Flow, Energy
Efficiency
\end{abstract}

Stirred vessels and bubble-column reactors have long been used in the chemical industry for gas-liquid agitation. Easier operation is commonly known for such conventional reactors aside from system efficiency. Recently, however, equipment embodying novel techniques such as the electrostatic smallbubble generator ${ }^{1-3)}$ and the orifice mixer ${ }^{4)}$ have been reported to produce as small bubbles as conventional equipment. In addition, the electrostatic method seemed to give better energy efficiency for poorly conductive liquid dielectrics. ${ }^{3,6)}$

In the present paper, the efficiency of the electrostatic bubble generator will be quantitatively compared with those of a stirred vessel ${ }^{5)}$ and an orifice mixer. ${ }^{4)}$

The bubble generation system is almost the same as the previous experiment. ${ }^{3)}$ Figure 1 shows an outline of the system. Five different liquid dielectrics shown in Table 1 were used as working fluid. Research grade $\mathrm{N}_{2}$ gas was injected into the liquids through a stainless steel hypodermic needle. Its inside and outside diameters were 0.27 and $0.51 \mathrm{~mm}$, respectively. The experiment was carried out in liquids of interest for applied $\mathrm{DC}$ voltage from 0 to $15 \mathrm{kV}$, gas flow rate from 0.4 to $1.0 \mathrm{~cm}^{3} / \mathrm{s}$, electrode gap distance from 1 to $8 \mathrm{~cm}$, specific electric conductivity from $10^{-12}$ to $10^{-5} \mathrm{~s} / \mathrm{m}$, and relative dielectric constant from 2 to 25 .

As it is defined in the previous paper, ${ }^{3)}$ the energy efficiency $\eta$ is given by

$$
\eta=\frac{6 \gamma M / \bar{d}_{B}}{W} \times 100[\%]
$$

Received September 24, 1983. Correspondence concerning this article should be addressed to S. Ogata, Laboratory of Marine Science, Faculty of Engineering, O University, Oita $870-11$. K. Tan is now with Sumitomo Chem. Co., Ltd., Nihama.

$$
W=P M+V I
$$

For the orifice mixer and the stirred vessel, $W$ becomes equal to $P M$.

Average bubble diameter $\bar{d}_{B}$ is shown in Fig. 2 as a function of $W / M$ and specific electric conductivity $\kappa$ for kerosene-ASA-3* mixtures. It can be seen that the electrostatic method is highly sensitive to conductivity since the dielectric constant of these mixtures remains almost constant despite the wide range of conductivity change. ${ }^{1)}$ Likewise, Fig. 3 shows the variation of $\eta$ as a function of $W / M$ and $\kappa$ for the same mixtures.

In the case of liquids with lower conductivity, $15 \mathrm{kV}$ applied voltage is still not sufficient for taking data over a wide range of $W / M$. It can be seen, however, that $\eta$ sharply falls with increasing $\kappa . \eta$ is expected to decrease with increasing applied electrical energy due to the generation of an electrohydrodynamic flow. ${ }^{6}$

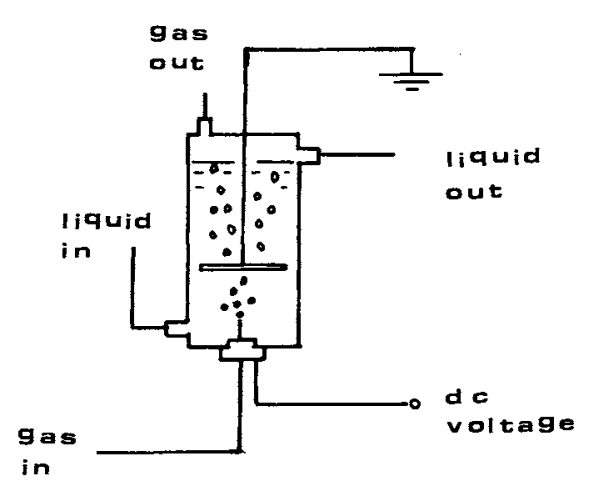

Fig. 1. Present version of bubble generation system.

* Anti-static agent: Shell Oil Co. ${ }^{1)}$ 